\title{
Acknowledgement to Reviewers of Hydrology in 2018
}

\author{
Hydrology Editorial Office \\ MDPI, St. Alban-Anlage 66, 4052 Basel, Switzerland \\ Published: 10 January 2019
}

Rigorous peer-review is the corner-stone of high-quality academic publishing. The editorial team greatly appreciates the reviewers who contributed their knowledge and expertise to the journal's editorial process over the past 12 months. In 2018, a total of 69 papers were published in the journal, with a median time to first decision of 15 days and a median time to publication of 44 days. The editors would like to express their sincere gratitude to the following reviewers for their cooperation and dedication in 2018:

Abbas, Azhar

Alexander, Malov

Al-Muqdadi, Sameh W.

Alvisi, Stefano

Amato, Daniel

Anagnostou, Marios

Apollonio, Ciro

Aschonitis, Vassilis

Ayalew Moges, Mamru

Ayele, Gebiaw T.

Bak, Lukasz

Barbetta, Silvia

Barbieri, Maurizio

Barloková, Danka

Bellos, Vasilis

Bergillos, Rafael J.

Bilskie, Matthew

Biudes, Marcelo S.

Bojar, Ana-Voica

Bosa, Silvia

Boschetti, Tiziano

Brambilla, Davide

Briciu, Andrei-Emil

Chau, Kwok-wing

Chen, Duan

Chen, Lu
Chen, Wei-Bo

Chen, Yi-Chung

Chen, Yong

Chiu, Long $S$

Chung, Eun-Sung

Chung, Sewoong

Croitoru, Adina-Eliza

Cugliari, Jairo

Davidson, Paul

De La Fuente, Alberto

De Lourdes Gomes Pereira, Maria

De Morais, Marcos Vinicius Bueno

De Paola, Pierfrancesco

Deb, Proloy

Delle Rose, Marco

Demetriou, Elias

Demirel, Mehmet

Denić-Jukić, Vesna

Dezetter, Alain

Di Giuseppe, Dario

Dietrich, Jörg

Dinis, Maria Alzira Pimenta

Djebou, Dagbegnon Clement Sohoulande

Du, Xinzhong

Dulai, Henrietta

Elci, Alper 
Elsayed, Saber M.

Emmer, Adam

Fang, Haiyan

Farjad, Babak

Feng, Ruimin

Fister, Iztok

Frappart, Frédéric

Ganguli, Poulomi

García-Lorenzo, Rafael

Gaume, Eric

Ge, Yingchun

Gilewski, Paweł Grzegorz

Gilleland, Eric

Gioia, Andrea

Giouri, Katerina

Gkiougkis, Ioannis

Grimaldi, Salvatore

Grimaldi, Stefania

$\mathrm{Gu}$, Huanghe

Guo, Qiaona

Guswa, Andrew

Güttler, Ivan

Ha, Lan Thanh

Haghighi, Ali Torabi

Hajigholizadeh, Mohammad

Hasan, Emad

Hejduk, Leszek

Hoghooghi, Nahal

Hoque, Shamia

Hsu, Shao Yiu

Huang, Guoru

Huang, Tianming

Hughes, Philip

Hunova, Iva

Ioana-Toroimac, Gabriela

Jafarzadegan, Keighobad

Jalbert, Jonathan

Jameel, Yusuf

Jang, Won Seok

Joshi, Sanjeev

Kanakoudis, Vasilis

Kapetas, Leon
Kazakis, Nerantzis

Kim, Jong Suk

Kim, Sang-Ug

Kim, Soojun

Kim, Sungwon

Kinnard, Christophe

Kirillin, Georgiy

Knight, Rodney

Kobayashi, Donald

Kong, Yanlong

Kooperman, Gabriel

Kostecki, Stanisław

Kotsopoulos, Spyros

Koutroulis, Aristeidis

Kumar, Pankaj

Kumar, Parveen

Kuo, Shu-lung

Laureano-Rosario, Abdiel E.

Li, Weide

Li, Yunliang

Li, Zhenhua

Lim, Kyoung-Jae

Liolios, Konstantinos A.

Liu, Haojie

Liu, Tie

Liu, Yaoze

Liu, Ying

Liu, Yonggang

Liu, Zhaowei

Lo, Kwong Fai A.

Löwe, Roland

Lu, Hui

Ma, Ronghua

Maggioni, Viviana

Manzano, Marisol

Marcinkowski, Pawel

Markert, Kel

Marrin, Donn L.

Maskey, Mahesh Lal

Matos Silva, Maria

Midttømme, Kirsti

Mirlohi, Susan 
Mleczko, Magdalena

Morsy, Mohamed

Mullens, Esther

Nakagawa, Kei

Nardi, Fernando

Nguyen, Van Khanh

$\mathrm{Nicu}$, Ionut Cristi

Niemitz, Jeffrey W.

Nobrega, Rodolfo

Noguchi, Kimihiro

Noh, Seong Jin

Núñez Cobo, Jorge Humberto

Obolewski, Krystian

Oehler, Till

Omani, Nina

Onishi, Kyosuke

Page, Declan

Palanisamy, Bakkiyalakshmi

Papatheodorou, Constantine

Parajka, Juraj

Park, Daeryong

Pasanisi, Francesco

Pereira, Luísa

Perera, Edangodage Duminda Pradeep

Pérez-Sánchez, Julio

Pires, Carlos

Pokhrel, Bijay K.

Porter, P. Steven

Prakash, Satya

Procesi, Monia

Ptak, Mariusz

Qin, Cheng-Zhi

Refice, Alberto

Reis, Mariane S.

Remenyi, Tomas

Rijal, Santosh

Ripa, Maria Nicolina

Rodrigo Comino, Jesús

Rodríguez-Blanco, M. Luz

Rodríguez-Solà, Raúl

Rohrer, Mario

Romanowicz, Renata
Rössler, Ole

Ruther, Nils

Sadeghian, Amir

Saifullah, Muhammad

Sangiorgio, Matteo

Saponieri, Alessandra

Sappa, Giuseppe

Schardong, Andre

Seo, Bong-Chul

Seo, Youngmin

Sharma, Suresh

Shen, Jianjian

Shi, Haiyun

Shiau, Jenq-Tzong

Shibata, Tomo

Shrestha, Maheswor

Shrestha, Narayan

Siddique, Ridwan

Sidiropoulos, Epaminondas

Sieczka, Anna

Silva, Ralph Trancoso Da

Simelton, Elisabeth

Simonescu, Claudia Maria

Singovszka, Eva

Son, Kyongho

Song, Lixiang

Song, Wenlong

Sordo-Ward, Alvaro

Sraj, Mojca

Stavi, Ilan

St-Hilaire, André

Sugimoto, Ryo

Tahvonen, Outi

Tallini, Marco

Tan, Jing

Teixido, Teresa

Tiranti, Davide

Tsoukalas, Ioannis A.

$\mathrm{Tu}$, Min-Cheng

Tyralis, Hristos

Uhl, Mathias

Urban, Thomas 
Varouchakis, Emmanouil A.

Vasiliades, Lampros

Vélez Upegui, Jorge Julián

Vigiak, Olga

Vogel, Kristin

Volpi, Elena

Walega, Andrzej

Wang, Chenghao

Wang, Fugang

Wang, Huimin

Wang, Tao

Wang, Weiwen

Wang, Xianwei

Ward, Timothy

Watson, Cameron Scott

Webb, Ryan W.

Williams, Gustavious Paul

$\mathrm{Wu}$, Guang-Zheng

Wu, Ming-Chang
$\mathrm{Wu}$, Wei

$\mathrm{Xu}$, Shiguo

Yan, Hongxiang

Yang, Xiaohua

Yannopoulos, Stavros

Yee, Susan

Yen, Haw

Yin, Zhenliang

You, Cheol Hwan

Younus, $\mathrm{Md}$

Zare, Soheil

Zeiger, Sean

Zeng, Yujin

Zhang, Jingxiong

Zhang, Xingnan

Zhao, Meng

Zhou, Ruijie

Zischg, Andreas Paul

(C) 2019 by the authors. Licensee MDPI, Basel, Switzerland. This article is an open access article distributed under the terms and conditions of the Creative Commons Attribution (CC BY) license (http://creativecommons.org/licenses/by/4.0/). 